\title{
Efectividad de la psicoterapia breve en los centros de salud mental II: Resultados.
}

\author{
Effectiveness of Brief Psychotherapy in Community Mental Health Centers II: Results.
}

\author{
Javier Fernández Méndez ${ }^{\text {a }}$, Marco A. Luengo Castro ${ }^{\text {b }}$, Juan García Haro ${ }^{c}$, Andrés Cabero Álva- \\ rez $^{\mathrm{d}}, \mathbf{M}^{\mathrm{a}}$ Dolores González Díaz ${ }^{\mathrm{e}}$, Begoña Braña Menéndez ${ }^{\mathrm{f}}$, Marcelino Cuesta Izquierdo ${ }^{\mathrm{g}}$, Pedro \\ José Caunedo Riesco ${ }^{\text {h. }}$
}

${ }^{a}$ Psicólogo Clínico. CSM II, La Calzada. Area V. Gijón. Servicio de Salud del Principado de Asturias (S.E.S.P.A.), ${ }^{b}$ Psicólogo Clínico. Unidad de Interconsulta y Enlace. Hospital Universitario Central de Asturias. Area IV. Oviedo. S.E.S.P.A., ${ }^{C} P$ sicólogo Clínico. CSM I. La Ería. Area IV. Oviedo. S.E.S.P.A., ${ }^{d}$ Psicólogo Clínico. CSM II, Teatinos. Area IV. Oviedo. S.E.S.P.A., ${ }^{e}$ Psicóloga Clínica. CSM IV, Pumarín. Area V.

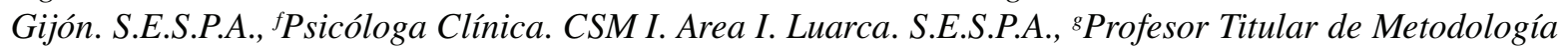
de las CC. del Comportamiento. Facultad de Psicología. Universidad de Oviedo., ${ }^{h}$ Psiquiatra. CSM I. Area I. Luarca. S.E.S.P.A.

Correspondencia: Javier Fernández Méndez (javierfermen@yahoo.es)

Recibido: 10/02/2010; aceptado con modificaciones: 29/06/2010

RESUMEN: Introducción. El objetivo de la presente investigación ha sido comparar mediante un ensayo clínico la efectividad de una Psicoterapia Breve con el tratamiento habitual de los Trastornos Mentales Comunes en los Centros de Salud Mental (CSM) de Asturias. La introducción general a este estudio se ha descrito por Fernández-Méndez y cols. (2010) y se remite a los lectores a ese artículo para más detalles respecto al fundamento, diseño, tratamientos y procedimientos de la investigación.

Sujetos y método. Se seleccionaron al azar 216 personas mayores de 14 años que consultaban por primera vez en seis CSM y que fueron diagnosticadas de trastornos depresivos, de ansiedad y/o de adaptación. Ciento cuarenta y una cumplían los criterios de inclusión y aceptaron participar en la investigación, siendo asignadas al azar a dos grupos: Psicoterapia breve integradora-ecléctica $(n=76)$ o Tratamiento habitual en los CSM $(n=65)$. Se han comparado sus resultados a los 6, 12, 24 y 36 meses en diversos índices de mejoría clínica, funcionamiento psicosocial e indicadores sanitarios indirectos. Los datos se han obtenido del Registro de Casos Psiquiátricos, de la Historia Clínica y del propio paciente. Se han usado los siguientes instrumentos: Impresión Clínica Global (ICG, Guy, 1976), Inventario de Discapacidad de Sheehan (SDI, Sheehan, 1996) y Cuestionario de Satisfacción (Moré y Muñoz, 2000).

Resultados. El programa de Psicoterapia breve ha sido más efectivo que el tratamiento habitual: Obtiene una mayor tasa de altas y los sujetos muestran
ABSTRACT: Background. The aim of the current research has been comparing the effectiveness of a brief psychotherapy with the usual treatment of Common Mental Disorders in Mental Health Centers (MHC) of Asturias. The general background of this study has already been described by FernándezMéndez et al (2010) and readers are referred to that article for details regarding rationale, design, treatments and procedures of the study.

Subjects and method. Two hundred and sixteen patients over the age of 14 were selected at random among those who consulted for the first time in six MHC and were diagnosed of depressive, anxiety or adjustment disorders. One hundred and forty-one fulfilled the inclusion criteria and agreed to take part in the study; they were assigned at random into two groups: brief integrative-eclectic psychotherapy $(n=76)$ or usual treatment in the MHC $(n=65)$. Their results have been compared at 6, 12, 24 and 36 months against diverse indexes of clinical improvement, psycho-social functioning and sanitary indirect indicators. Information has been obtained from the Psychiatric Cases Record, the Clinical History and from the patients. The following instruments have been used: the Clinical Global Impression of Improvement scale, the Sheehan Disability Inventory and a Satisfaction Survey.

Results. Brief psychotherapy was more effective than usual treatment: it resulted in a higher number of discharges and subjects show greater improvements in clinical status and disability, and greater 
ORIGINALES Y REVISIONES

mayores mejorías en estado clínico y discapacidad y mayor satisfacción. Estas diferencias se dan tanto a los seis meses como al año y a los dos años de iniciarse los tratamientos. Además, aunque el número medio de sesiones es igual en ambos grupos (en torno a seis), el tratamiento experimental duró mucho menos tiempo. Conclusiones: La Psicoterapia ha resultado ser un tratamiento viable y efectivo para la mayoría de los casos que consultan en los CSM.

PALABRAS CLAVE: Psicoterapia breve, Psicoterapia de corta duración, Trastornos mentales comunes, Trastornos mentales leves, Ensayo clínico, Efectividad, Centros de Salud Mental, Servicios de Salud Mental. satisfaction. These differences occur both at 6 months to one year and two years of starting treatment. Furthermore, although the average number of sessions is equal in both groups (about six), time-wise the psychological treatment lasted much less.

Conclusions: Psychotherapy has proven a viable and effective treatment for most cases consulted at the MHC.

KEY WORDS: Brief Psychotherapy, Short-term Psychotherapy, Common Mental Disorders, Minor Psychiatric Disorders, Clinical trial, Effectiveness, Community Mental Health Centers, Mental Health Services.

\section{AGRADECIMIENTOS}

Esta investigación ha sido financiada por los Servicios de Salud Mental del Principado de Asturias.

Los autores desean agradecer la colaboración de la Unidad de Coordinación de Salud Mental, de los coordinadores y compañeros de los Centros de Salud Mental (especialmente las profesionales encargadas de gestionar las demandas), de la Biblioteca Rodríguez Lafora de Salud Mental y de los pacientes que aceptaron participar.

En el presente artículo se informa de los resultados de un estudio en el que se comparó la efectividad de una Psicoterapia breve con el tratamiento habitual de personas diagnosticadas de Trastornos Mentales Comunes atendidas en seis Centros de Salud Mental (CSM) de Asturias. A continuación se presentan las principales características del estudio. Para una descripción detallada del diseño de investigación y los tratamientos aplicados consultar Fernandez-Méndez y cols (1).

Entre los pacientes derivados por los cauces habituales a los CSM participantes se seleccionaron 141 sujetos que cumplían los siguientes criterios de inclusión: Ser mayor de 14 años; acudir por primera vez a ese CSM; no ser diagnosticado de trastornos mentales severos; no haber tenido un intento autolítico grave en los seis meses anteriores; requerir atención especializada; acudir al menos a una consulta en el CSM; y aceptar participar en la investigación. Los participantes fueron asigandos al azar a los dos grupos de tratamiento: 76 al grupo experimental y 65 al control, que constituyen la muestra de intención de tratamiento.

Los pacientes recibieron dos tipos de tratamiento: Los asignados al grupo control, las intervenciones que se utilizan habitualmente en los CSM del Principado de Asturias; y los asignados al experimental, un programa de terapia breve cuyo componente fundamental es la terapia psicológica, que se ha aplicado siguiendo una guía de tratamiento con un modelo integrador-ecléctico. En el grupo experimental el $82 \%$ de los participantes recibió psicoterapia, combinada con psicofármacos en 
ORIGINALES Y REVISIONES

la mitad de los casos, y el 12\% sólo tratamiento farmacológico. Los pacientes de este grupo tuvieron una media de siete sesiones en seis meses. Y en el grupo de tratamiento habitual recibieron psicofármacos el $86 \%$ de los sujetos, combinados con psicoterapia en una quinta parte de los casos y el 3\% recibió sólo psicoterapia. Tuvieron unas 7 sesiones en 14 meses. Los terapeutas del grupo de terapia breve fueron seis psicólogos clínicos y un psiquiatra con formación y experiencia en psicoterapia y los del grupo control 6 psicólogos clínicos y 20 psiquiatras.

Los sujetos fueron evaluados a los 6, 12, 24 y 36 meses del inicio del tratamiento en diversas variables:

- Resultado clínico entendido como estatus respecto al tratamiento en el CSM: si continúa a tratamiento, fué dado de alta, abandonó y/o volvió a solicitar tratamiento posteriormente.

- Estado clínico: Escala de cambio o mejoría de la Impresión Clínica Global (Clinical Global Impression, CGI-GI, 2) según el juicio del paciente.

- Grado de discapacidad según el paciente valorado con el Inventario de Discapacidad de Sheehan (Sheehan Disability Inventory, SDI, 3).

- Satisfacción del usuario respecto al tratamiento, evaluada mediante determinadas preguntas de un Cuestionario de Satisfacción (4).

- Indice de Recuperación. Consideramos casos recuperados los que no recibían ningún tipo de tratamiento en Salud Mental o Atención Primaria ni habían tenido bajas laborales por motivos de salud mental en los últimos 6-12 meses.

Los resultados de cada momento temporal se presentan en las tablas 2, 3, 4 y 5. Además, para las principales variables de resultado se representan gráficamente los resultados desde el punto de vista longitudinal (figuras 1-5).

\section{Características de la muestra.}

La muestra está compuesta por 141 personas cuyas características se recogen en la tabla 1. Resultó formada básicamente los siguientes grupos diagnósticos: Trastornos de adaptación (F43.2), Trastornos depresivos sin síntomas psicóticos (F32-F39) y Trastornos neuróticos, secundarios a situaciones estresantes y somatomorfos (F40-F49). También se podían admitir personas sin trastorno mental que sin embargo requieren atención especilizada (diagnosticables según el eje III de la CIE-10: "Factores que influyen en el estado de salud y en el contacto con los servicios de salud"), pero no hubo ningún caso entre los participantes (y uno solo entre los perdidos). La muestra estudiada está constituida entonces por los llamados Trastornos Mentales Comunes (4). No hemos hecho una evaluación que nos permita establecer su severidad inicial, pero cabe suponer que se trata de casos de 
ORIGINALES Y REVISIONES

severidad leve-moderada: incluimos casos que no son severos a priori pero que ya han sido derivados al nivel especializado, es decir, que no han podido resolverse en AP, y en ese sentido no serían meramente leves. Además, presentaban síntomas desde hacía unos seis meses (mediana) y una cuarta parte tenía antecedentes de tratamiento especializado (ya fuera en el ámbito privado o en otros CSM).

Tabla 1

Descripción de la muestra estudiada y comparación de los grupos en variables pretratamiento. Variables socio-demográficas.

\begin{tabular}{|c|c|c|c|c|}
\hline Variable & Total & Experimental & Control & Resultados \\
\hline Edad & 39.83 & $\begin{array}{l}\text { Media: } 36.80 \\
\text { Mediana: } 36.5 \\
\text { Rango: } 19-63\end{array}$ & $\begin{array}{l}43.37 \\
43.00 \\
16-75\end{array}$ & $\begin{array}{c}\mathrm{t}(139)=-3.087 \\
\mathrm{p}=0.002\end{array}$ \\
\hline Sexo & $\begin{array}{l}\mathrm{V}(38.3) \\
\mathrm{M}(61.7)\end{array}$ & $\begin{array}{l}\mathrm{V}(36.8 \%) \\
\mathrm{M}(63.2 \%)\end{array}$ & $\begin{array}{l}\mathrm{V}(40.0 \%) \\
\mathrm{M}(60.0 \%)\end{array}$ & $\begin{array}{c}\mathrm{x}^{2}(1)=0.148 \\
p=0.701\end{array}$ \\
\hline $\begin{array}{l}\text { Estado civil. } \\
\qquad \begin{array}{l}\text { - Solteros: } \\
\text { - Casados: } \\
\text { - Viudos: } \\
\text { - Divorc: } \\
\text { - Convive pareja: }\end{array}\end{array}$ & $\begin{array}{c}38(27.0 \%) \\
81(57.4) \\
5(3.5) \\
9(6.4) \\
8(5.7)\end{array}$ & $\begin{array}{c}24(31.6) \\
41(53.9) \\
3(3.9) \\
5(6.6) \\
3(3.9)\end{array}$ & $\begin{array}{c}14(21.5) \\
40(61.5) \\
2(3.1) \\
4(6.2) \\
5(7.7)\end{array}$ & $\begin{array}{l}\text { Usando variable agrupada (3): } \\
\qquad \begin{array}{c}\mathrm{x}^{2}(1)=1.934 \\
p=0.164\end{array}\end{array}$ \\
\hline $\begin{array}{l}\text { Convivencia. } \\
\text { - Fam origen: } \\
\text { - Fam propia: } \\
\text { - Con otros: } \\
\text { - Solo: }\end{array}$ & $\begin{array}{c}30(21.3) \\
95(67.4) \\
2(1.4) \\
14(9.9)\end{array}$ & $\begin{array}{l}21(27.6) \\
49(64.5) \\
1(1.3) \\
5(6.6)\end{array}$ & $\begin{array}{l}9(13.8) \\
46(70.8) \\
1(1.5) \\
9(13.8)\end{array}$ & $\begin{array}{c}\text { (eliminado categoría "con otros") } \\
\mathrm{x}^{2}(2)=5.200 \\
p=0.074\end{array}$ \\
\hline Con hijos a su cargo. & Si: 61 (43.3) & SI: 34 (44.7) & SI: 27 (41.5) & $\begin{array}{c}\mathrm{x}^{2}(1)=0.046 \\
p=0.702\end{array}$ \\
\hline $\mathrm{N}^{\mathrm{o}}$ de hijos a cargo. & 0.67 & 0.75 & 0.58 & $\begin{array}{c}\mathrm{t}(138)=1.102 \\
p=0.273\end{array}$ \\
\hline $\begin{array}{l}\text { Nivel instrucción: } \\
\text { - Sin estudios: } \\
\text { - Primarios: } \\
\text { - Secundarios: } \\
\text { - Universitarios: }\end{array}$ & $\begin{array}{c}2(1.4) \\
64(45.7) \\
52(37.1) \\
22(15.7)\end{array}$ & $\begin{array}{c}0(0.0) \\
29(38.7) \\
30(40.0) \\
16(21.3)\end{array}$ & $\begin{array}{c}2(3.1) \\
35(53.8) \\
22(33.8) \\
6(9.2)\end{array}$ & $\begin{array}{l}\text { Agrupando datos (4) } \\
\qquad \begin{array}{c}\mathrm{x}^{2}(2)=6.063 \\
p=0.048\end{array}\end{array}$ \\
\hline $\begin{array}{l}\text { Situación laboral: } \\
\text { - Trabajando: } \\
\text { - Parado: } \\
\text { - Jubilado: } \\
\text { - Ama casa: } \\
\text { - Incapacidad transitoria (I.T.) }\end{array}$ & $\begin{array}{l}50(35.5) \\
13(9.2) \\
15(10.6) \\
20(14.2) \\
35(24.8)\end{array}$ & $\begin{array}{c}29(38.2) \\
6(7.9) \\
6(7.9) \\
12(15.8) \\
17(22.4)\end{array}$ & $\begin{array}{l}21(32.3) \\
7(10.8) \\
9(13.8) \\
8(12.3) \\
18(27.7)\end{array}$ & $\begin{array}{c}\text { (limitándonos a dos categorías: } \\
\text { trabajando / I.T.) } \\
\mathrm{x}^{2}(1)=0.737 \\
p=0.391\end{array}$ \\
\hline Medio de residencia urbano $(\mathrm{n}, \%)$ & $75(53.2)$ & $42(55.3)$ & $33(50.8)$ & $\begin{array}{c}\mathrm{x}^{2}(1)=0.284 \\
p=0.594\end{array}$ \\
\hline
\end{tabular}


Tabla 1 (CONTINUACIÓN)

Variables clínicas.

\begin{tabular}{|c|c|c|c|c|}
\hline Variable & Total & Experimental & Control & Resultados (1) \\
\hline $\begin{array}{c}\text { Tipo de consulta: } \\
\text { - Ordinaria. } \\
\text { - Preferente. }\end{array}$ & $\begin{array}{c}127(90.1) \\
14(9.9)\end{array}$ & $\begin{array}{l}68(89.5) \\
8(10.5)\end{array}$ & $\begin{array}{c}59(90.8) \\
6(9.2)\end{array}$ & $\begin{array}{c}\mathrm{x}^{2}(1)=0.066 \\
\quad p=0.798\end{array}$ \\
\hline $\begin{array}{l}\text { Anteceds. Asistenciales: } \\
\begin{array}{l}\text { - M.AP } \\
\text { - Especialistas. } \\
\text { - Sin antecedentes. }\end{array}\end{array}$ & $\begin{array}{l}41(29.1) \\
35(24.8) \\
65(46.1)\end{array}$ & $\begin{array}{l}21(27.6) \\
18(23.7) \\
37(48.7)\end{array}$ & $\begin{array}{l}20(30.8) \\
17(26.1) \\
28(43.1)\end{array}$ & $\begin{array}{c}\mathrm{x}^{2}(3)=0.459 \\
\quad p=0.928\end{array}$ \\
\hline $\begin{array}{l}2 \text { o más consultas con } \\
\text { MAP en año previo. }\end{array}$ & $\begin{array}{c}62 \text { de } 117 \\
(53.0 \%)\end{array}$ & $\begin{array}{l}27 \text { de } 60 \\
(45.0 \%) \\
\end{array}$ & $\begin{array}{l}35 \text { de } 57 \\
(61.4 \%) \\
\end{array}$ & $\begin{array}{c}\mathrm{x}^{2}(1)=3.157 \\
\quad p=0.076\end{array}$ \\
\hline $\begin{array}{l}\mathrm{N}^{\circ} \text { consultas con MAP } \\
\text { en año anterior. }\end{array}$ & $\begin{array}{c}\mathrm{N}=117 \\
2.12\end{array}$ & $\begin{array}{c}\mathrm{N}=60 \\
\text { Media: } 2.03\end{array}$ & $\begin{array}{c}\mathrm{N}=57 \\
2.21\end{array}$ & $\begin{array}{c}\mathrm{t}(115)=-0.588 \\
p=0.558\end{array}$ \\
\hline $\begin{array}{l}\text { Con tratamiento fármacológico } \\
\text { previo. }\end{array}$ & $\begin{array}{c}76 \text { de } 141 \\
53.9 \%\end{array}$ & $\begin{array}{c}\text { Si: } 39 \text { de } 76 \\
51.3 \% \\
\end{array}$ & $\begin{array}{c}\text { Si: } 37 \text { de } 65 \\
56.9 \%\end{array}$ & $\begin{array}{c}\mathrm{x}^{2}(1)=0.443 \\
\quad p=0.506\end{array}$ \\
\hline $\begin{array}{l}\text { Tiempo con síntomas } \\
\text { (meses; media, mediana) }\end{array}$ & $\begin{array}{c}16.6 \\
6.0\end{array}$ & $\begin{array}{c}18.8 \\
7.0\end{array}$ & $\begin{array}{c}14.0 \\
6.0\end{array}$ & $\begin{array}{c}\mathrm{t}(138)=1.029 \\
p=0.305\end{array}$ \\
\hline $\begin{array}{l}\text { Motivo consulta: } \\
\text { - Problemas.(2) } \\
\text { - Inicio sintomas. } \\
\text { - Descompensación. } \\
\text { - Auto-hetero agresividad. }\end{array}$ & $\begin{array}{l}30(21.4) \\
58(41.4) \\
50(35.7) \\
2(1.4)\end{array}$ & $\begin{array}{c}20(26.3) \\
34(44.7) \\
21(27.6) \\
1(1.3)\end{array}$ & $\begin{array}{l}10(15.6) \\
24(37.5) \\
29(45.3) \\
1(1.6) \\
\end{array}$ & $\begin{array}{c}\text { (limitándonos a dos categorías: } \\
\text { inicio / descompensación) } \\
\mathrm{x}^{2}(1)=2.968 \\
p=0.085\end{array}$ \\
\hline $\begin{array}{l}\text { Grupos diagnósticos } \\
\qquad \begin{array}{l}\text { - T. Depresivos. } \\
\text { - T. Adaptativos. } \\
\text { - T. de Ansiedad. } \\
\text { - Otros: } \\
\text { • T. de Personalidad. } \\
\text { - T. Control impulsos. } \\
\text { • Disfunción sexual. }\end{array}\end{array}$ & $\begin{array}{c}44(31.2) \\
56(39.7) \\
34(24.1) \\
7(5.0) \\
2 \\
3 \\
2\end{array}$ & $\begin{array}{c}20(26.3) \\
33(43.4) \\
19(25.0) \\
4(5.3) \\
1 \\
2 \\
1\end{array}$ & $\begin{array}{c}24(36.9) \\
23(35.4) \\
15(23.1) \\
3(4.6) \\
1 \\
1 \\
1\end{array}$ & $\begin{array}{c}\text { (sin usar la categoría “otros”) } \\
\mathrm{x}^{2}(2)=1.884 \\
p=0.390\end{array}$ \\
\hline
\end{tabular}

Notas: (1) Para las variables cuantitativas la prueba estadística utilizada ha sido la Prueba T para la igualdad de medias en muestras independientes y para variables nominales la chi-cuadrado $\left(\mathrm{x}^{2}\right)$; (2) Problemas de relación social, problemas familiares, problemas laborales y solicitud de orientación y/o consejo; (3) Por un lado casados o viviendo en pareja; por otro, solteros, divorciados y viudos; (4) En tres categorías: sin estudios o con estudios primarios, con estudios secundarios, y con estudios universitarios.

Como puede verse en la tabla 1 , hemos comparado los dos grupos en 16 variables pre-tratamiento. De ellas encontramos diferencias significativas $(\mathrm{p}<0.05)$ 
ORIGINALES Y REVISIONES

en dos: edad y nivel de instrucción. Estas diferencias, según nuestros análisis de casos incluidos y perdidos, pueden deberse a una combinación de causas, entre ellas, diferencias dadas por azar y diferencias entre los sujetos que aceptaron/rechazaron participar en cada grupo. Dado que esas variables podrían sesgar los resultados hemos investigado su efecto, comprobando que los resultados no varían significativamente. Pero sí consideramos que nuestro estudio no es generalizable al subgrupo de mayor edad (mayores de 64 años). En cualquier caso, el efecto de las variables pre-tratamiento será objeto de un informe posterior.

\section{A los seis meses (ver tabla 2).}

Resultado clínico. Se refiere a las proporciones de casos que fueron dados de alta por su terapeuta (por no requerir más tratamiento por parte del CSM), los que abandonaron y los que siguen a tratamiento. A los 6 meses, en el grupo de psicoterapia breve habían sido dados de alta más sujetos (46.1\%) que en el grupo de tratamiento habitual (24.6\%), siendo la diferencia estadísticamente significativa $(p=0.008)$. El grupo de psicoterapia breve tuvo más abandonos que el grupo de tratamiento habitual $(25.0 \%$ y $15.4 \%$ respectivamente), aunque la diferencia no es estadísticamente significativa. Lógicamente, a los 6 meses siguen muchos más sujetos a tratamiento en el grupo de tratamiento habitual (el 58.5\%), que en el de psicoterapia breve (el 28,9\%).

Estado clínico según el paciente. En la ICG, el grupo experimental supera significativamente $(p=0.004)$ al control en el porcentaje de sujetos que dicen estar mucho o bastante mejor que antes del tratamiento (43.4\% vs. $12.9 \%)$. No hay diferencias significativas en los que dicen estar solo algo mejor, igual o peor, aunque todas las tasas son favorables para el grupo experimental.

Grado de discapacidad según el paciente. En comparación con los del tratamiento habitual, los pacientes evaluados del grupo de psicoterapia informaron de menor discapacidad en la escala global del SDI $(p=0.008)$.

Al considerar estas comparaciones (ICG y SDI) deben tenerse en cuenta dos cuestiones: (1) que en la evaluación de seguimiento con ICG y SDI participaron 84 sujetos de los 141 participantes, y (2), que de las personas que participaron en esta evaluación seguían a tratamiento en el CSM el $34.0 \%$ de los del grupo experimental y el $61.3 \%$ del control.

Satisfacción con el tratamiento. Evaluada generalmente al mes del alta o abandono o a los 6 meses si la persona seguía a tratamiento. Para el análisis de resultados hemos agrupado las categorías de respuesta. Como se puede observar en la tabla 5, hay más sujetos que dicen estar satisfechos o muy satisfechos con 
el tratamiento en el grupo experimental (94\%) que en el control (70\%), siendo la diferencia estadísticamente significativa.

Tabla 2

Comparación entre los tratamientos a los 6 meses.

\begin{tabular}{|c|c|c|c|c|c|}
\hline Variable & Experimental & Control & $\begin{array}{l}\text { Estadístico } \\
\text { de contraste }\end{array}$ & $\begin{array}{l}\text { Valor } \\
\text { de } p\end{array}$ & $\begin{array}{l}\text { Tamaño de } \\
\text { efecto (6) }\end{array}$ \\
\hline Altas (n, \%) (1) & $35(46.1 \%)$ & $16(24.6 \%)$ & $x^{2}(1)=6.974$ & 0.008 & $V=0.222$ \\
\hline Abandonos (n, \%) (1) & $19(25.0 \%)$ & $10(15.4 \%)$ & $\mathrm{x}^{2}(1)=1.983$ & 0.159 & $V=0.119$ \\
\hline $\begin{array}{l}\text { Siguen a tratamiento en CSM } \\
(\mathrm{n}, \%)(1)\end{array}$ & $22(28.9 \%)$ & $38(58.5 \%)$ & $x^{2}(1)=12.484$ & 0.000 & $V=0.298$ \\
\hline $\begin{array}{l}\text { ICG (2) } \\
\text { - Mucho o bastante mejor } \\
\text { - Algo mejor } \\
\text { - Igual } \\
\text { - Peor }\end{array}$ & $\begin{array}{c}43.4 \\
43.4 \\
11.3 \\
1.9\end{array}$ & $\begin{array}{c}12.9 \\
54.8 \\
25.8 \\
6.5\end{array}$ & $\begin{array}{c}\mathrm{x}^{2}(1)=8.338 \\
1.027 \\
2.955 \\
1.183\end{array}$ & $\begin{array}{l}0.004 \\
0.311 \\
0.086 \\
0.277\end{array}$ & $V=0.315$ \\
\hline Discapacidad global (0-30) (2) & 13.36 & 17.97 & $T(82)=-2.718$ & 0.008 & $R=0.288$ \\
\hline $\begin{array}{l}\text { Satisfacción 7: Cree que las } \\
\text { consultas han sido beneficiosas (5): } \\
\text { - Muy de acuerdo. } \\
\text { - De acuerdo. } \\
\text { - En desacuerdo. }\end{array}$ & $\begin{array}{c}21(31.3 \%) \\
42(62.7 \%) \\
4(6.0 \%)\end{array}$ & $\begin{array}{l}17(27.9 \%) \\
25(41.0 \%) \\
19(31.1 \%)\end{array}$ & $x^{2}(2)=14.267$ & 0.001 & $V=0.334$ \\
\hline $\begin{array}{l}\text { Satisfacción 10: Valoración global } \\
\text { de la ayuda recibida (5): } \\
\text { - Muy satisfactoria. } \\
\text { - Satisfactoria. } \\
\text { - Insatisfactoria. }\end{array}$ & $\begin{array}{c}18(26.9 \%) \\
45(67.2 \%) \\
4(6.0 \%)\end{array}$ & $\begin{array}{l}11(18.0 \%) \\
32(52.5 \%) \\
18(29.5 \%)\end{array}$ & $x^{2}(2)=12.540$ & 0.002 & $V=0.313$ \\
\hline
\end{tabular}

Notas: (1) Sobre la muestra total de cada grupo (76/65). (2) Sobre 84 casos, 53 del experimental y 31 del control; en evaluación realizada a los 5.0 (media) - 6.0 (mediana) meses de iniciar el tratamiento.. (3) Sobre 120 casos, 63 del experimental y 57 del control. (4) Sobre 107 casos, 57 del experimental y 50 del control. (5) Sobre 128 casos, 67 del experimental y 61 del control. (6) $V$ de Cramer o $R$ de Pearson.

\section{Al año (ver tabla 3).}

Resultado clínico. A los 12 meses, la proporción de altas en el grupo de psicoterapia breve alcanza el $56.6 \%$, mientras que en grupo de tratamiento habitual llega hasta el $33.8 \%$, siendo la diferencia estadísticamente significativa $(p=0.007)$. La diferencia en la tasa de abandonos disminuye (28.9\% frente a $20.0 \%$ ) y no es estadísticamente significativa. De nuevo hay más sujetos que siguen a tratamiento en grupo de intervenciones habituales $(44,6 \%)$ que en el grupo de psicoterapia breve $(14,5 \%)$. 
ORIGINALES Y REVISIONES

Estado clínico según el paciente. No hay diferencias estadísticamente significativas entre los grupos. El grupo experimental tiene más sujetos que dicen estar mucho o bastante mejor ( $55.2 \%$ vs. $33.3 \%$ ), pero menos que dicen estar solo algo mejor $(24.1 \%$ vs. $42.9 \%)$.

Grado de discapacidad según el paciente. La diferencia en discapacidad global resulta significativa $(p<0.05)$, siendo menor en el grupo experimental.

Tasas de recuperación. Las tasas de recuperación son significativamente superiores en el grupo experimental (65.5 vs. 23.7\%).

$\mathrm{Al}$ considerar las comparaciones en ICG y SDI debe tenerse en cuenta la pérdida de casos y que, de las personas que participaron en esta evaluación $(n=50)$ sólo seguían a tratamiento en el CSM el 10.3\% de los del grupo experimental, mientras que continuaban el $47.3 \%$ del control.

Tabla 3

Comparación entre los tratamientos al año.

\begin{tabular}{|l|c|c|c|c|c|}
\hline \multicolumn{1}{|c|}{ Variable } & Experimental & Control & $\begin{array}{c}\text { Estadístico } \\
\text { de contraste }\end{array}$ & $\begin{array}{c}\text { Valor } \\
\text { de } \mathrm{p}\end{array}$ & $\begin{array}{c}\text { Tamaño de } \\
\text { efecto }\end{array}$ \\
\hline Altas (n, \%) (1) & $43(56.6 \%)$ & $22(33.8 \%)$ & $\mathrm{x}^{2}(1)=7.287$ & 0.007 & V Cramer $=0.227$ \\
\hline Abandonos (n, \%) (1) & $22(28.9 \%)$ & $13(20 \%)$ & $\mathrm{x}^{2}(1)=1.503$ & 0.220 & $V=0.103$ \\
\hline $\begin{array}{l}\text { Siguen a tratamiento en CSM } \\
\text { (n, \%) (1) }\end{array}$ & $11(14.5 \%)$ & $29(44.6 \%)$ & $\mathrm{x}^{2}(1)=15.664$ & 0.000 & $V=0.333$ \\
\hline ICG (2) & & & $\mathrm{x}^{2}(1)=$ & & \\
$\quad$ - Mucho o bastante mejor & 55.2 & 33.3 & 3.339 & 0.126 & \\
$\quad$ - Algo mejor & 24.1 & 42.9 & 1.961 & 0.161 & \\
$\quad$ - Igual & 10.3 & 19.0 & 0.766 & 0.381 & \\
$\quad$ - Peor & 10.3 & 4.8 & 0.516 & 0.473 & \\
\hline Discapacidad global (0-30)(2) & 10.76 & 16.38 & $\mathrm{~T}(48)=-2.319$ & 0.025 & $R=0.317$ \\
\hline Sujetos recuperados (n, \%)(3) & $38(65.5)$ & $14(23.7)$ & $\mathrm{x}^{2}(1)=20.685$ & 0.000 & $V=0.420$ \\
\hline
\end{tabular}

Notas: (1) Sobre la muestra total de cada grupo (76 / 65). (2) Sobre 50 casos, 29 del experimental y 21 del control; según evaluación realizada a los 9.6 (media) - 10.0 (mediana) meses de iniciar el tratamiento. (3) Sobre 117 casos, 58 del experimental y 59 del control.

A los dos años (ver tabla 4).

Resultado clínico. A los 2 años se mantiene una mayor tasa de altas en el grupo de psicoterapia, incluso aumentando la diferencia (67.1\% vs. 49.2\%), que es significativa $(p=0.032)$. En cambio, la tasa de abandonos prácticamente se iguala $(28.9 \%$ vs. $27.7 \%$ ). En este plazo, siguen a tratamiento en el CSM desde el principio el $3.9 \%$ de los sujetos del grupo experimental y el $21.5 \%$ del grupo control. 
Estado clínico según el paciente. En el grupo experimental hay más casos que dicen estar mucho o bastante mejor ( $82.6 \%$ vs. $43.8 \%)$ y menos que refieren estar igual (0.0\% vs. 21.9.\%). Ambas diferencias son significativas $(p<0.05)$. En los que dicen estar solo algo mejor y los que dicen estar peor no hay diferencias significativas.

Grado de discapacidad según el paciente. Se mantiene la diferencia significativa a favor del grupo de psicoterapia breve en discapacidad global $(p=0.032)$.

Tasas de recuperación. Las tasas de recuperación son significativamente superiores en el grupo experimental (66.7 vs. 40.4\%).

De nuevo, al valorar las comparaciones en ICG y SDI debe tenerse en cuenta la pérdida de casos y que, de las personas que participaron en esta evaluación $(n=52)$, ninguno de los del grupo experimental seguía a tratamiento en el CSM mientras que sí lo hacían el $20.7 \%$ de los que participaron del grupo control.

Retorno. A los dos años apenas hay diferencias en la tasa de retornos entre los dos grupos (9.6\% vs. 10.0\%). Pero si a los retornos agregamos los que seguían a tratamiento desde el principio, tenemos que a los 2 años el $13.2 \%$ de los sujetos del grupo experimental y el $29.7 \%$ del control estaban a tratamiento en el CSM. La diferencia es estadísticamente significativa $(p=0.016)$.

Tabla 4

Comparación entre los tratamientos a los 2 años.

\begin{tabular}{|l|c|c|c|c|c|}
\hline \multicolumn{1}{|c|}{ Variable } & Experimental & Control & $\begin{array}{c}\text { Estadístico } \\
\text { de contraste }\end{array}$ & $\begin{array}{c}\text { Valor } \\
\text { de } \mathrm{p}\end{array}$ & $\begin{array}{c}\text { Tamaño de } \\
\text { efecto }\end{array}$ \\
\hline Altas (n, \%) (1) & $51(67.1 \%)$ & $32(49.2 \%)$ & $\mathrm{x}^{2}(1)=4.623$ & 0.032 & V Cramer $=0.181$ \\
\hline Abandonos (n, \%) (1) & $22(28.9 \%)$ & $18(27.7 \%)$ & $\mathrm{x}^{2}(1)=0.27$ & 0.869 & $V=0.14$ \\
\hline $\begin{array}{l}\text { Siguen a tratamiento en CSM } \\
\text { (n, \%) (1) }\end{array}$ & $3(3.9 \%)$ & $14(21.5 \%)$ & $\mathrm{x}^{2}(1)=10.225$ & 0.001 & $V=0.269$ \\
\hline ICG (2) & & & $\mathrm{x}^{2}(1)=$ & & \\
$\quad$ - Mucho o bastante mejor & $82.6 \%$ & $43.8 \%$ & 8.419 & 0.004 & $V=0.391$ \\
$\quad$ - Algo mejor & $17.4 \%$ & $25.0 \%$ & 0.454 & 0.50 & \\
$\quad$ - Igual & $0 \%$ & $21.9 \%$ & 5.765 & 0.016 & $V=0.324$ \\
- Peor & $0 \%$ & $9.4 \%$ & 2.281 & 0.131 & \\
\hline Discapacidad global (0-30) (2) & 6.00 & 10.97 & $\mathrm{~T}(54)=-2.201$ & 0.032 & $R=0.287$ \\
\hline Sujetos recuperados (n, \%) (3) & $38(66.7)$ & $23(40.4)$ & $\mathrm{x}^{2}(1)=7.934$ & 0.005 & $V=0.264$ \\
\hline Retorno global (4) & $7(9.6 \%)$ & $5(10.0 \%)$ & $\mathrm{x}^{2}(1)=0.006$ & 0.940 & \\
\hline Sujetos a tto. en CSM (5) (6) & $10(13.2 \%)$ & $19(29.7 \%)$ & $\mathrm{x}^{2}(1)=5.780$ & 0.016 & $V=0.203$ \\
\hline
\end{tabular}

Notas: (1) Sobre la muestra total de cada grupo (76 / 65). (2) Sobre 55 casos, 23 del experimental y 32 del control; según evaluación realizada a los 23.7 (media) - 24.0 (mediana) meses de iniciar el tratamiento. (3) Sobre 114 casos, 57 del experimental y $57 \mathrm{del}$ control (4) sobre 123 casos, 73 del experimental y 50 del control. (5) sobre 140 casos, 76 del experimental y 64 del control. (6) Porque siguieron o retornaron. 
ORIGINALES Y REVISIONES

A los tres años (ver tabla 5).

Resultado clínico. A los 3 años continúa la diferencia empírica en la tasa de altas, el grupo control llega a tener mayor tasa de abandonos, y se mantienen a tratamiento más casos en el grupo de tratamiento habitual. Pero ninguna de estas diferencias es estadísticamente significativa.

Tasas de recuperación. No hay diferencias significativas en las tasas de sujetos objetivamente recuperados (62.5 vs. $47.3 \%$ ), aunque sean mayores en el grupo experimental.

Retorno. Tampoco en este plazo se encuentran diferencias en la proporción de retornos (12.2\% vs. 12.7\%). Aunque los sujetos del grupo control tardan más tiempo en volver al CSM (13.0 vs. 16.7 meses), la diferencia no es significativa. Si a los retornos añadimos los que siguieron a tratamiento desde el inicio, tenemos que a los 3 años están a tratamiento en el CSM 11 sujetos (14.5\%) del experimental y $16(25.0 \%)$ del control. La diferencia no es estadísticamente significativa.

Tabla 5

Comparación entre los tratamientos a los 3 años.

\begin{tabular}{|l|c|c|c|c|c|}
\hline \multicolumn{1}{|c|}{ Variable } & Experimental & Control & $\begin{array}{c}\text { Estadístico } \\
\text { de contraste }\left(\mathrm{x}^{2}\right)\end{array}$ & $\begin{array}{c}\text { Valor } \\
\text { de } \mathrm{p}\end{array}$ & $\begin{array}{c}\text { Tamaño de efecto } \\
(\mathrm{V} \text { de Cramer) }\end{array}$ \\
\hline Altas (n, \%) (1) & $51(67.1 \%)$ & $34(52.3 \%)$ & $3.204(1)$ & 0.073 & 0.151 \\
\hline Abandonos (n, \%) (1) & $23(30.3)$ & $21(32.3)$ & $0.068(1)$ & 0.79 & 0.022 \\
\hline Siguen a tratamiento (n, \%) (1) & $2(2.6)$ & $9(13.8)$ & $6.126(1)$ & 0.013 & 0.208 \\
\hline Recuperados (n, \%) (2) & $35(62.5)$ & $26(47.3)$ & $2.599(1)$ & 0.107 & 0.153 \\
\hline $\begin{array}{l}\text { Retorno (solo de altas y } \\
\text { abandonos) (3) }\end{array}$ & $9(12.2 \%)$ & $7(12.7 \%)$ & $0.009(1)$ & 0.92 & 0.008 \\
\hline Sujetos a tto. en CSM (4) (5) & $11(14.5 \%)$ & $16(25.0 \%)$ & $2.473(1)$ & 0.116 & 0.133 \\
\hline
\end{tabular}

Notas: (1) Sobre la muestra total de cada grupo (76 / 65). (2) Sobre 111 casos, 56 del experimental y 55 del control. (3) sobre 129 casos, 74 del experimental y 55 del control. (4) sobre 140 casos, 76 del experimental y 64 del control. (5) porque siguieron o retornaron.

\section{Otras variables.}

Número de sesiones y duración del tratamiento. Debido a la heterogeneidad de los resultados intragrupo, informamos de la mediana y un percentil obtenidos en estas variables (ver tabla 2 de nuestro anterior artículo, 1). La mitad de los casos asignados al programa de psicoterapia breve asistieron a 6 sesiones o menos con un facultativo y el $75 \%$ recibieron 7 sesiones o menos (rango 1-26). Y de los asignados al tratamiento habitual, el 50\% tuvieron 6 y el $75 \% 9$ sesiones o menos (rango 1-29). 
ORIGINALES Y REVISIONES

La duración del tratamiento en el CSM fue mucho mayor en el grupo control (287 días $=9.4$ meses $)$ que en el de terapia breve (124 días $=4.1$ meses $)$.

Analizando el grupo experimental, encontramos que 39 (51.3\% de grupo) sujetos fueron dados de alta tras 8 sesiones o menos; 33 (49.2\% de los 67 evaluados en el grupo) dijeron estar mucho o bastante mejor tras 7 sesiones o menos; y 31 (54.4\% de 57 evaluados) estaban recuperados después de 7 sesiones o menos. Por tanto, para que la mitad de los componentes del grupo alcanzaran una mejoría significativa fueron suficientes menos de 8 sesiones.

Resultados de las altas en la primera evaluación de seguimiento. En la evaluación realizada al mes de la última consulta a la que acudieron, los que fueron dados de alta en el grupo experimental dicen encontrarse mejor $(69.7 \%$ vs. $25.0 \%$ de bastante o mucho mejor; $\chi 2(1)=7.207, p=0.007$ ), refieren menor discapacidad global (11.47 vs. 17.33, t $(44)=-2.039, p=0.048)$ y están más satisfechos que los del grupo control (97.8\% vs. $79.0 \%$ de satisfechos o muy satisfechos). El control resulta favorecido en la tasa de pacientes que están sólo algo mejor (21.2\% vs. 58.3\%). En estas dos comparaciones (satisfacción e ICG “algo mejor”) no se puede aplicar prueba estadística por carecer de suficientes casos. No hubo diferencias en ICG igual o peor. Resultados de los abandonos. En la evaluación realizada a los cinco meses de la última consulta a la que acudieron, entre los que abandonaron las consultas en el CSM, no hay diferencias entre los grupos en el grado de mejoría (ICG) ni en satisfacción con el tratamiento $(85.7 \%$ y $75.0 \%$ de satisfechos o muy satisfechos con terapia breve y con tratamiento habitual respectivamente); pero los del grupo de psicoterapia refieren menor discapacidad global (14.2 vs. 21.5; t (18) = -2.133, $p=0.047$ ). Globalmente, el $45.0 \%$ de los sujetos que abandonaron dijeron encontrarse bastante o mucho mejor y el $80.5 \%$ dijeron estar satisfechos o muy satisfechos con el tratamiento.

Resultados de los que completaron los tratamientos. Completaron los tratamientos (asistieron al menos a tres consultas o a menos si se les dió el alta facultativa) 127 sujetos, el 90.1\% de los que los iniciaron: 71 (93.4\%) en el grupo experimental y 56 $(86.2 \%)$ en el control. Esos pacientes obtienen los mismos resultados que las personas que los iniciaron y no los completaron. En ninguna de las variables de resultado encontramos diferencias significativas entre ambas muestras.

\section{Discusión y conclusiones:}

Nuestro objetivo era estudiar en un contexto real de servicios públicos de Salud Mental la efectividad de la psicoterapia con una metodología de investigación lo más rigurosa posible y un tamaño muestral significativo.

Desde el punto de vista metodológico, esta investigación tiene una elevada vali- 
ORIGINALES Y REVISIONES

dez externa, pero en parte a costa de una disminución relativa de la validez interna. La validez externa queda respaldada, entre otros aspectos, por la participación de pacientes extraídos de los que solicitaron consulta en los CSM por los cauces habituales, por el número de participantes, por la amplitud de los criterios de inclusión (que implica una heterogeneidad de los sujetos en cuanto a diagnóstico y otras variables, variabilidad buscada con el objetivo de sacar conclusiones sobre la mayoría de las demandas en los CSM -cumplieron los criterios de inclusión el 83.3\% de los casos nuevos- y no sobre casos muy específicos; asimismo se admite la comorbilidad, que es relativamente frecuente en los TMC), por la utilización de criterios de resultado ligados a la práctica clínica, por haberse realizado en las condiciones habituales de trabajo en los CSM (especialmente representativas en lo que se refiere al grupo control), por contar con terapeutas que trabajan en tales servicios y por aplicar tratamientos igualmente representativos de lo habitual en condiciones reales (y esto no sólo el "tratamiento habitual", sino también el experimental: el uso de una guía de tratamiento ha proporcionado mayor estructuración a la terapia de lo usual, pero dejando suficiente margen de maniobra para que los terapeutas trabajaran según su modelo-estilo y las necesidades de los pacientes concretos, como de hecho hacen en contextos reales). Además, hemos contrastado el efecto específico de dos posibles amenazas a la validez externa: La derivada de aquellos sujetos que, cumpliendo criterios de inclusión, no aceptaron participar en la investigación y la debida a la pérdida de sujetos en las evaluaciones de seguimiento con la ICG y la SDI. Respecto a la primera tenemos una sobrerrepresentación de sujetos que residen en medio rural respecto al urbano y de consultas ordinarias respecto a las preferentes. En cuanto a la segunda, de nuevo predominan las personas de medio urbano entre los perdidos y en general hay una pérdida importante de casos que restaría validez a las conclusiones basadas en el ICG y la SDI, pero también hemos comprobado que los participantes en esas evaluaciones no se diferencian significativamente en variables críticas de los que no participaron. Sin duda estas consideraciones deben tenerse en cuenta a la hora de generalizar los resultados pero creemos que no cuestionan seriamente su generalizabilidad.

La validez interna ha descansado fundamentalmente en la aleatorización, en el uso de diversas fuentes de información para recoger datos sobre las variables de resultado y en la participación de colaboradores ajenos al equipo de investigación en la asignación de los casos a los tratamientos y en la recogida de datos. Pero el diseño presenta debilidades en este aspecto de la validez. Tenemos por un lado que algunos aspectos pensados para favorecer la validez externa perjudican la interna: la heterogeneidad de los sujetos participantes, la carencia de medidas pre-tratamiento (debida a la necesidad de que el grupo control fuera ciego para que representara efectivamente el "tratamiento habitual") y la variabilidad de los tratamientos (que supone una cierta indefinición de la variable independiente). También atentan contra la validez interna otras características del diseño debidas fundamentalmente a limitaciones de recursos 
ORIGINALES Y REVISIONES

y tiempo: el uso de autoinformes sencillos (sin grandes garantías psicométricas), no haber asegurado la fiabilidad de los diagnósticos (nos hemos limitado al juicio clínico del facultativo responsable del caso, sin usar, por ejemplo, una entrevista estructurada), y no haber comprobado la adherencia de los terapeutas y pacientes a los tratamientos. En relación con la validez interna, hemos comprobado específicamente el posible efecto de diferencias previas entre los sujetos asignados a los tratamientos. Creemos que no es significativo, pero nos obliga a ser cautelosos en nuestras conclusiones; por ejemplo, consideramos que nuestros resultados no son válidos para pacientes mayores de 64 años.

También debe tenerse en cuenta que el tratamiento experimental se ve favorecido porque tanto los consultantes como los terapeutas sabían desde el principio que estaban participando en una investigación, lo que no era el caso en el grupo control. En cambio, el grupo control se ve favorecido por un mejor ajuste entre el caso y el tratamiento. Los casos se suelen distribuir entre los clínicos de los CSM de acuerdo a qué terapeuta / tratamiento se considera más apropiado; los casos del experimental no pasaron por esa selección previa de forma que en este grupo se incluyen algunos casos que en realidad sólo fueron abordables mediante tratamiento farmacológico y no recibieron psicoterapia.

En fin, a nuestro diseño se le puede cuestionar en algún grado la validez interna, teniendo como punto fuerte la validez externa. Es lógico según nuestro objetivo de realizar un estudio de efectividad en el contexto real de asistencia pública, pues para asegurar la validez interna y la eficacia ya existen numerosos ensayos clínicos aleatorizados.

Teniendo en cuenta estas consideraciones, recapitulamos a continuación los principales resultados:

1. A los seis meses, en el grupo de psicoterapia breve se obtiene una mayor mejoría clínica, tanto desde el punto de vista de los terapeutas (porcentaje de altas) como desde la perspectiva de los pacientes (estado clínico y discapacidad).

2. Al año se mantiene la superioridad del grupo de psicoterapia en el porcentaje de sujetos dados de alta y en discapacidad global, no habiendo diferencias en el grado de mejoría referido por los pacientes. Además, el grupo de terapia breve tiene relativamente más sujetos recuperados.

3. A los dos años, el grupo de terapia breve ha alcanzado una mayor tasa de altas y los pacientes informan de mayor mejoría clínica y menor discapacidad. Se mantiene la superioridad del grupo experimental en la tasa de recuperación.

4. A los tres años se iguala la tasa de altas y la proporción de sujetos recuperados. No disponemos de evaluaciones del estado clínico de los pacientes en ese plazo.

5. En el grupo de terapia breve resultan relativamente más pacientes satisfechos con el tratamiento que en el grupo de tratamiento habitual.

6. No se observan diferencias significativas en las tasas de abandono, aunque a 
ORIGINALES Y REVISIONES

los seis meses y al año son mayores en el grupo de terapia breve. Tampoco hay diferencias en el estado clínico y la satisfacción entre los abandonos de ambos grupos. Sí las hay en discapacidad, que es menor en los abandonos del grupo de terapia breve. Cabe destacar que casi la mitad de los pacientes que abandonaron dijeron encontrarse bastante o mucho mejor en las evaluaciones de seguimiento -resultado por lo demás acorde con otras investigaciones al respecto $(5,6)-$, y el $80.5 \%$ estaban satisfechos con el tratamiento. Por tanto, abandono no significa mal resultado.

7. Las tasas de alta dependen fundamentalmente del juicio de las terapeutas que puede ser sesgado. Sin embargo, comprobamos que coincide con el juicio que los pacientes hacen sobre estado clínico, grado de discapacidad y satisfacción con el tratamiento. En esos tres índices el grupo de terapia breve supera al tratamiento habitual cuando se consideran solo los sujetos dados de alta.

8. No hay diferencias significativas entre los dos grupos en el porcentaje de sujetos que, tras ser dados de alta o haber abandonado, han retornado al CSM a los dos $\mathrm{y}$ tres años del inicio del tratamiento original.

En definitiva, el programa de terapia breve ha sido más efectivo que el tratamiento habitual en los CSM en tres momentos temporales (a los 6, 12 y 24 meses), igualándose a los 3 años. Y este resultado ha sido consistente en nuestros tres tipos de criterios (el del terapeuta, el del paciente y los indicadores de recuperación). Desde el punto de vista estadístico las diferencias no son grandes. Los tamaños de efecto (de altas, ICG, SDI y recuperación) se sitúan, según el tipo de variable, entre 0.181 y 0.420 (V de Cramer) y entre 0.287 y 0.317 ( $\mathrm{R}$ de Pearson) y son, por tanto, relativamente pequeños. Sin embargo, desde la perspectiva clínica y práctica las diferencias (como se ve en las gráficas $1,3,4$ y 5) pueden considerarse importantes.

No hemos hallado diferencias entre la muestra de intención de tratamiento y la de los que completaron los tratamientos. En otras investigaciones los resultados de los que completan la terapia son mejores que los de la muestra de intención de tratamiento, debido a una mayor adherencia a los tratamientos. Creemos que en la nuestra no ocurre por un motivo: el número de sesiones no está predeterminado, sino que depende tanto de la adherencia como del resultado clínico. Así, los que recibieron pocas sesiones pueden ser los casos que más mejoraron y/o los que abandonaron y los que recibieron muchas pueden ser los que menos respondieron y/o tenían más adherencia al tratamiento.

Debemos destacar que el mantenimiento en el tiempo de diferencias a favor del grupo experimental se da a pesar de que en el grupo de tratamiento habitual los pacientes continúan mucho más tiempo a tratamiento en el CSM (1). Los resultados a los dos años y las tasas de retorno a los dos y tres años son especialmente importantes porque pueden indicar que los efectos de la psicoterapia breve se mantienen en el tiempo (cuando se evaluó a los pacientes a los dos años, hacía más de un año que el $80 \%$ había terminado el tratamiento en el CSM). De todas maneras, la evolución postratamiento 
Figura 1. Evolución de las tasas de alta.

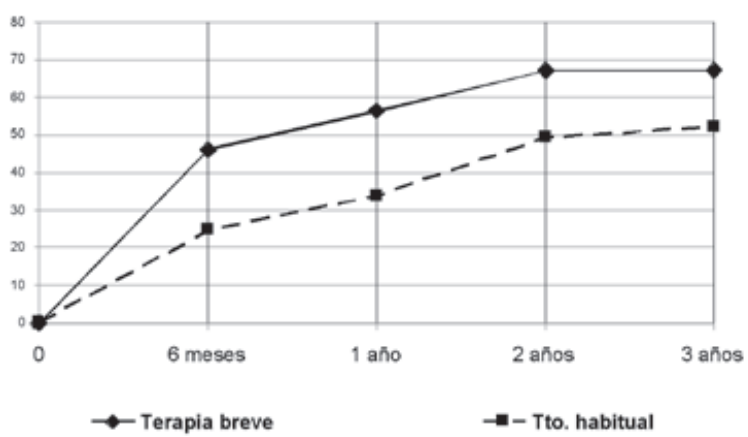

Figura 3. Porcentaje bastante o mucho mejor (ICG).

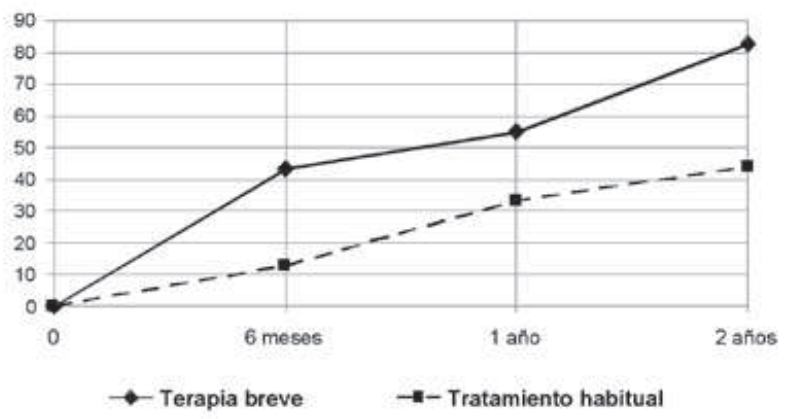

Figura 2. Evolución de las tasas de abandono.

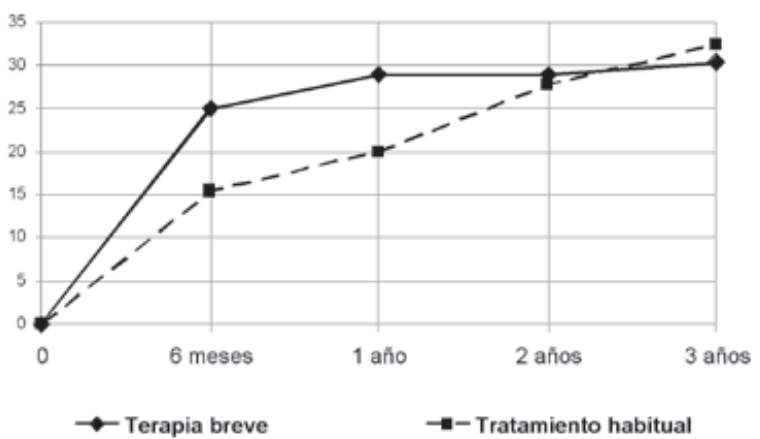

Figura 4. Evolución del resultado en discapacidad global (SDI, el punto inicial es arbitrario).

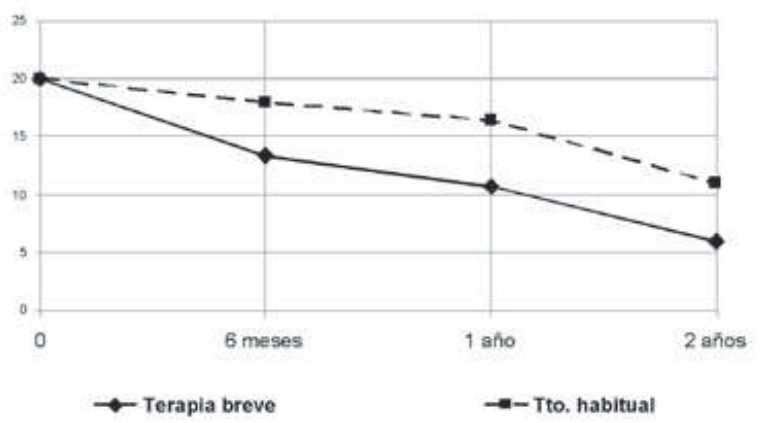

Figura 5. Evolución de las tasas de recuperación.

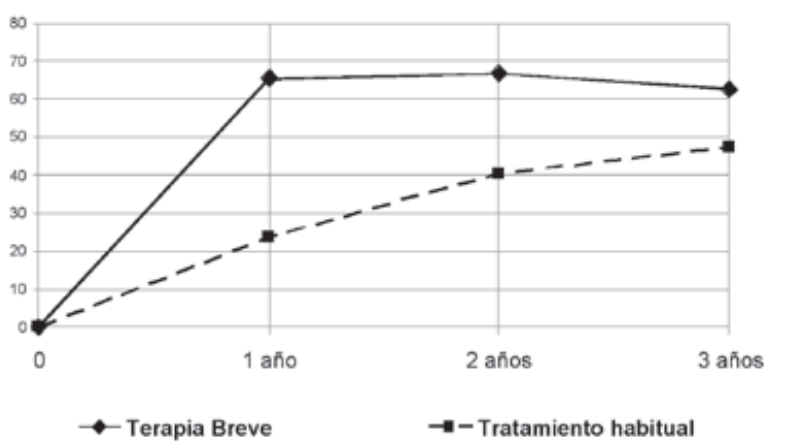

de los pacientes será objeto de un análisis detallado en una publicación ulterior.

El tratamiento psicoterapéutico no sólo se ha mostrado efectivo, sino también viable en nuestros servicios teniendo en cuenta el exceso de demanda en relación con los recursos disponibles. El número de sesiones se sitúa en torno a seis y el $75 \%$ de los casos asignados al grupo de psicoterapia han recibido siete sesiones o menos, siendo las primeras de 45 minutos y las siguientes de 30 . Como indican las sesiones de los dados de alta, que refirieron mejoría o que consideramos recuperados, este tratamien- 
ORIGINALES Y REVISIONES

to puede conseguir una mejoría clínica significativa en la mitad de los pacientes con menos de 8 sesiones, cifra inferior a las 13-18 sesiones que se requieren en los ensayos clínicos según Hansen, Lambert y Forman (7). Este aspecto es importante pues a veces se intenta justificar la escasez de oferta de psicoterapia en los servicios públicos alegando que es un tratamiento demasiado exigente en cuanto a consumo de recursos. Nuestros resultados indican el tratamiento psicológico no solo es efectivo, sino asumible por nuestros servicios para una cantidad elevada de pacientes.

Por otro lado, y apuntando ya a la eficiencia, aunque no hay diferencias entre los grupos en el número de sesiones, la duración de tratamiento en el CSM es muy superior en el grupo control (una mediana de 287 días frente a 124 del grupo de psicoterapia breve, 1), tal vez por tener un mayor intervalo entre sesiones. Actualmente estamos realizando un análisis de los costes asociados a ambos tratamientos del que se informará oportunamente.

En este momento se encuentra en proceso de análisis de resultados la que ha sido la segunda fase de este proyecto de investigación. Se ha llevado a cabo con la idea de aumentar la validez interna, controlando mejor dos fuentes de sesgo: la de las posibles diferencias previas entre los sujetos asignados a los grupos y el hecho de que el grupo control fuera ciego y el experimental no. En esta segunda fase se ha hecho una evaluación psicométrica antes del tratamiento y tanto los terapeutas como los pacientes de ambos grupos sabían desde el inicio de su participación en la investigación.

Tal vez el aspecto más controvertido de nuestra investigación sea el hecho de que los tratamientos aplicados no sean puros, es decir, que no hemos comparado dos tratamientos específicos sino dos modelos de trabajo: el tratamiento habitual, en el que predominan los fármacos y sólo un pequeño porcentaje de pacientes recibe psicoterapia y un modelo en el que la mayoría de los pacientes recibe psicoterapia y a la mitad se le proporciona además tratamiento farmacológico (de hecho, los que tomaron fármacos ya los tenían prescritos desde AP; de no ser así, tal vez los hubieran tomado muchos menos pacientes). En cualquier caso, ya que la mitad de la muestra tomaba fármacos antes de iniciar el tratamiento en el CSM, no podemos responder a la pregunta de si en general es mejor como primera opción de tratamiento en los Trastornos Mentales Comunes la psicoterapia o los fármacos. Ahora bien, si nos atenemos al nivel de atención especializada, nuestro estudio sí puede acercarse a la cuestión: si los médicos de AP ya utilizan habitualmente fármacos en el tratamiento de estos trastornos y derivan a los CSM los casos que con sus herramientas no pueden resolver, ¿qué es lo que deben aportar los CSM? Nuestros datos indican que proporcionar psicoterapia puede ser más efectivo que prescribir más fármacos. De todas maneras, es necesaria la realización de investigaciones en las que se compare claramente la efectividad de la psicoterapia y los fármacos solos y en combinación en condiciones reales y con una metodología más rigurosa que la que hemos podido desarrollar en el presente estudio.

Como conclusión, podemos decir que la psicoterapia es un tratamiento viable en 
los Centros de Salud Mental y, posiblemente, más efectivo que el tratamiento habitual, casi exclusivamente farmacológico.

\section{BIBLIOGRAFÍA:}

(1) Fernández-Méndez J, Luengo MA, García-Haro J, Cabero A, González-Díaz MD, Braña B, Cuesta M, Caunedo PJ. Efectividad de la psicoterapia breve en los Centros de Salud Mental I: Diseño, tratamientos y procedimiento. Rev Asoc Esp Neurpsiq. 2010; 30 (108), 563-580.

(2) GuY W. Early Clinical Drug Evaluation (ECDEU) Assessment Manual. Rockville: National Institute of Mental Health, 1976.

(3) Sheehan DV, Harnett-Sheehan K, Raj BA. The measurement of disability. Int Clin Psychopharmacol, 1996; 11 (Suppl 3): 89-95.

(4) Moré MA, MuÑoz PE. Satisfacción de los usuarios de un servicio de salud mental. Archivos de Psiquiatría, 2000; 63: 139-158.

(5) PекARIK G. Psychotherapy abbreviation. A practical guide. New York: The Haworth Press, 1996.

(6) Bados A, García Grau E, Fusté A. Eficacia y utilidad de la terapia psicológica. Revista Internacional de psicología Clínica y de la Salud, 2002; 2 (3): 477-502

(7) Hansen NB, LAMbert MJ, Forman EM. The Psychotherapy dose-response effect and its implications for tratment delivery services. Clinical Psychology: science and practice. 2002; 9(3): 329-343. 